Article

\title{
The Potential of Three Computer-Based Communication Activities for Supporting Older Adult Independent Living
}

\author{
Melinda Heinz ${ }^{1, *}$, Jinmyoung Cho ${ }^{2}$, Norene Kelly ${ }^{3}$, Peter Martin ${ }^{3}$, Johnny Wong ${ }^{4}$, \\ Warren Franke ${ }^{5}$, Wen-Hua Hsieh ${ }^{3}$ and Joan Blaser ${ }^{3}$ \\ 1 Department of Psychology, Upper Iowa University, Fayette, IA 52142, USA \\ 2 Baylor Scott \& White Health, Temple, TX 76508, USA; Jinmyoung.Cho@BSWHealth.org \\ 3 Department of Human Development and Family Studies, Iowa State University, Ames, IA 50011, USA; \\ nbkelly@iastate.edu (N.K.); pxmartin@iastate.edu (P.M.); wenhua@gmail.com (W.-H.H.); \\ jbaenzig55@gmail.com (J.B.) \\ 4 Department of Computer Science, Iowa State University, Ames, IA 50011, USA; wong@iastate.edu \\ 5 Department of Kinesiology, Iowa State University, Ames, IA 50011, USA; wfranke@iastate.edu \\ * Correspondence: heinzm@uiu.edu; Tel.: +1-563-425-5912
}

Academic Editor: Anna Fensel

Received: 27 April 2016; Accepted: 18 May 2016; Published: 24 May 2016

\begin{abstract}
Technology has become an increasingly integral part of life. For example, technology allows individuals to stay in touch with loved ones, obtain medical services through telehealthcare, and enjoy an overall higher quality of life. Particularly for older adults, using technology increases the likelihood that they will maintain their independence and autonomy. Long-distance caregiving has recently become a feasible option where caregivers for older adults can access reports and information about their loved one's patterns that day (e.g., food and medication intake). Technology may be able to offset age-related challenges (e.g., caregiving, accessing healthcare, decreased social networks) by applying technology to the needs of older adults. Solutions for meeting such challenges, however, have been less targeted. In addition, the healthcare system is evolving to focus on providing options and services in the home. This has direct implications for older adults, as the majority of healthcare services are utilized by older adults. Research is still at the beginning stages of developing successful technology tools that are compatible with older adult users. Therefore, the design, implementation, and outcome of such computer-based communication activities will be discussed in this paper in order to guide future endeavors in technology marketed for older adults.
\end{abstract}

Keywords: computer-based communication activities; technology; daily health diary; Skype; focus group

\section{Introduction}

\subsection{Technology and Older Adults}

As pointed out in [1], we are currently witnessing two remarkable trends: the rapid diffusion of technology and widespread population aging. Increasingly, technology has the potential to enhance older adults' abilities, relationships, and health. Compared to younger people, older people are less likely to adopt new technologies into their lives unless they see clear benefits to themselves [2]. Yet older adults are more willing to use technology than stereotypes suggest, and important predictors of adoption are attitude, nature of the experience, available support, and perceived utility [3]. Regardless of users' ages, existing research stresses the importance of a user-driven perspective 
in designing relevant, user-friendly technology [4-6]. Thus, the development and prototype of an innovation is aimed at older people needs to take into account all these factors.

A 2004 report from the National Research Council found that technology is typically developed by younger people for the use of younger people and marketed at younger target groups [7]. Similarly, it was noted in [8] that "[i]n the vast panoply of human-factors research that goes into design and marketing of modern technology, older users seldom make an appearance" (p. 68). Even when older adults are included in the development and design process, technology designers are still challenged to understand the sensory and cognitive effects that accompany aging [9]. In addition, the physical (e.g., arthritis) and cognitive changes (e.g., declining cognitive processing) of aging may affect ways in which older adults are able to use technology [10].

\subsection{Technology and Safety}

One example of opportunities presented by current technology is the ability of older adults to easily alert or check-in with professional staff or family. Older adults who do not check in may signal cause for concern and warrant further investigation from family or staff members. In particular, living alone is one of the greatest risk factors for older adults [11]. It was pointed out in [12] that falls posed societal and financial difficulties for individuals and the health care system at large. Technology applications may be able to alleviate some of the risk factors associated with living alone by simulating virtual contact through check-in measures.

It is also possible that older adults may feel more secure knowing that someone would check in with them if they failed to do so. This may also increase the likelihood that older adults would be able to remain independent in their own homes. For example, previous research has noted that older adult falls were linked to the loss of independence and autonomy [13]. If staff or family members were able to intervene more quickly, they may be able to render aid and assistance after a fall.

Although some devices allow users to indicate a current problem or emergency, other innovations can address the difficult-to-perceive yet critical effect of gradual decline. As pointed out in [14], change is a red flag in geriatric healthcare and is suggestive of the need for prompt investigation and action. They consequently called for research that helps determine which of today's technologies best support, motivate, and track healthy behaviors at home. Contemporary developments in science and technology are creating an evolutionary shift from clinic-centric to community-centric healthcare, placing health and wellness into the hands of an informed, proactive citizenry [14]. Healthy aging, therefore, "may evolve into a more holistic process through which optimal function and life quality are achieved using an array of health-related technologies that augment human interaction and support" ([14], p. 180). Thus, something like a daily health diary can play a vital role in this healthcare development. In terms of the design process, "small-scale outcomes studies of experiential prototypes" (p. 203) before larger studies with more thoroughly developed systems are contemplated have been recommended [14].

\subsection{The Advent of Computer Tools}

Tools such as a daily check-in or health diary, as well as currently available communication technologies such as Skype, have the potential to engender a broad positive impact in both individual lives and society. A daily check-in can ensure that older adult users will not be left incapacitated for a prolonged time; a health diary has the potential for early detection of serious problems; and Skype adds important visual information to traditional voice-only communication technology. Such advances support an older adult's ability to live independently, likely increasing both the quality of the person's life as well as the length of time the person can live outside the costly realm of professional care. Independence is a critical issue for many older adults, with their safety and health at risk as they try to cope with age-related issues [4,15]. Preventing or delaying the loss of independence should be a goal of people who care for older adults [15]. A Swedish study demonstrated considerable cost savings as well as benefits to older people and caregivers with the implementation of a home-based support system that used information and communication technology [5]. 
It was asserted in [4] that the diffusion of such innovations "begins with early adopters who are willing to explore new possibilities and risk trial use, and to lay the groundwork for others to follow" (p. 89). Meanwhile, the shortcomings of existing applied technologies lie in user compliance and effectiveness, cost and dependability, and sustainability and acceptance $[4,16]$. It was emphasized in [17] that one of the biggest research challenges of proactive health systems, especially for aging in place, was usability issues. Furthermore, three issues identified in [18] as impeding technology use by older adults are design factors, training issues, and users' awareness of potential benefits (or lack thereof). Given the previous findings, many technologies have supported older adults' independence, enhanced connection with family members or health care providers, and expanded their proactive roles in health systems; however, there are still challenges associated with older adults and healthcare professionals deriving benefits from technology tools. Thus, the purpose of the current study is to describe the set-up and installation of three computer-based communication tools for older adults and report on compliance and feedback related to such tools.

\section{Method}

\subsection{Participants}

After obtaining approval from the institutional review board at Iowa State University, participants were recruited from an independent living retirement community located in a university town. To be eligible for the study, participants had to be 70 years or older, possess a personal computer, have access to an Internet connection, and have basic computer skills. Eight older adults (three women and five men; Table 1) met the eligibility requirements and elected to participate in the study. Testing new computer tools is generally not feasible on a large scale because it requires significant hands-on time; we therefore included a relatively small sample of participants who would provide us with valuable information on the feasibility of the tools. Furthermore, previous research has indicated that sample sizes of four or five participants sufficiently yield information regarding usability and user experience concerns [19].

Table 1. Characteristics of participants $(n=8)$.

\begin{tabular}{cccc}
\hline \multicolumn{2}{c}{ Category } & Frequency & Percent \\
\hline \multirow{2}{*}{ Age $(\mathrm{M}=85.13, \mathrm{SD}=3.09)$} & Male & 5 & \\
\multirow{2}{*}{ Gender } & Female & 3 & 62.5 \\
& Total & 8 & 37.5 \\
& White & 8 & 100.0 \\
\hline Ethnicity & Married & 6 & 100.0 \\
\hline \multirow{2}{*}{ Marital Status } & Widowed & 2 & 75.0 \\
& Total & 8 & 25.0 \\
\hline \multirow{2}{*}{ Employment Status } & Retired & 8 & 100.0 \\
\hline
\end{tabular}

\subsection{Implementation of the Daily Diary and Skype}

An interdisciplinary team of researchers including Gerontology, Human Development and Family Studies, Computer Science, and Kinesiology departments built a technology tool useful for tracking older adult health. After the Computer Science department completed the design process, research assistants tested the product for errors before implementing it in the independent living apartment complex.

\subsection{Installation}

Research assistants installed separate, easily accessible icons (i.e., one icon for the check-in, a second icon for the daily diary, and a third icon for Skype (version 4.2) prominently on the desktop of 
the participants' computers to simplify the process. Therefore, unnecessary procedures and steps were eliminated, making the process more seamless and efficient. This was done to encourage compliance and reduce confusion or forgetfulness. Participants were also given written instructions on how to click on the separate icons in case they needed another reminder. In some cases, exchanging computers was necessary in order to make the computer-based activities fully functional. One participant had no additional disk space on his computer and thus needed to borrow a laptop, while another had a dial-up Internet connection, making the initial set-up cumbersome. In addition, several participants borrowed web cameras to participate in the Skype portion of the study since they did not possess their own. There were relatively few technical failures. In one instance, a participant had to attempt the Skype call again. However, no significant technical failures were reported.

\subsection{Procedure and Compliance}

Participants were assigned an ID and were asked to engage in a total of three computer-based communication activities during the ten-day period between the hours of $10 \mathrm{a} . \mathrm{m}$. and $12 \mathrm{p} . \mathrm{m}$.: (1) check-in with university researchers; (2) answer questions in a daily health diary; and (3) interact with research assistants via Skype. Task frequency, compliance, and answering questions for each participant were collected from all three tasks and entered into SPSS version 19 (IBM, Armonk, NY, USA). Following the ten-day period, a focus group session was conducted with participants. The purpose behind asking participants to engage in three tasks everyday was to assess how successful older adults were with following these instructions in order to simulate what it might be like to remotely monitor and interact with older adults from afar (i.e., demonstrating what it might be like for older adults to call in with professional healthcare workers, caregivers, or family members).

\subsubsection{Check-in}

The check-in was the simplest task in which the older adults participated. Older adults simply had to click on the check-in icon and type in their ID. Participants knew they had been successful with this step after they received a cartoon and a message that stated "Thanks for visiting us, have a wonderful day and see you tomorrow".

\subsubsection{Daily Health Diary}

The daily health diary consisted of 21 questions across five domains (e.g., computer questions, overall perceived health, nutritional consumption, physical activity, and cognitive patterns). The complete list of 21 questions were chosen to simulate what it might be like for participants to complete electronic monitoring information to submit to healthcare professionals. The included questions were chosen and presented on a daily basis to provide a thorough view of participant health and to assess daily changes. Participants had the opportunity to review their answers before they officially submitted their answers. After submitting their answers, a new window opened that indicated their answers had been submitted successfully. Given that the focus of this paper is more about the implementation of computer-based communication activities, specific information regarding items and scoring in the daily diary is not included.

\subsubsection{Skype Chat}

The final task for participants to complete each day was a Skype chat with research assistants. Between the hours of 10 a.m. and 12 p.m. participants were asked to call in. This task served as another assignment to assess the success of a virtual face-to-face check-in device. It also served to simulate the benefits and challenges of virtual contact with older adults.

\subsection{Focus Group Discussion}

After the ten days were completed, participants were invited to participate in a focus group to discuss their thoughts and opinions regarding the interventions. The focus group was conducted at the independent living apartment complex. All individuals engaged in a focus group after the completion of the ten-day period. Table 2 presents a complete list of questions asked at the focus group. The focus 
group was open-ended in nature and lasted for approximately one hour. Discussion from the focus group was videotaped and later transcribed verbatim. The transcripts were coded and categorized by two different researchers. As is common with qualitative research, a peer review was completed when researchers shared their initial coding and categorization with the rest of the research team [20]. Detailed descriptions and examples of each theme are discussed in the results section.

Table 2. Focus group questions.

General Questions

1. What was your overall experience participating in this study? What did you like? What did you not like?

2. What was it like to have someone come to your home for software and equipment installation?

Check-In Task

3. What was your experience like with the "check in" assignment?

4. What difficulties did you encounter conducting this task?

5. How much time did it take every day to complete this task?

6. Would you be interested in using the check-in assignment every day, if doctors and nurses are available to review your information and provide you with recommendations? Skype

7. What was your experience of using Skype?

a What do you like about using Skype?

b What do you dislike about using Skype?

c Did you encounter any difficulties?

d How did you feel about checking in with us on Skype every day?

e How much time did it take every day to complete this task?

f Do you think you might use Skype in the future? Why or why not?

$\mathrm{g}$ Would you be interested in using Skype every day, if doctors and nurses are available to review your information and provide you with recommendations?

Daily Health Diary

8. What was your experience of visiting the website with the health diary questions?

a What was it like answering the health diary questionnaire every day?

b Any recommendation on how we can improve the website? (e.g., appearance, questions)?

c What was the greatest difficulty you encountered conducting this task?

d How much time did it take every day to complete this task?

e Would you be interested in using the healthy diary every day, if doctors and nurses are available to review your information and provide you with recommendations?

9. What are the major factors that affect whether you want or don't want to use these applications?

10. How easy or difficult was it for you to remember to incorporate Skype and the daily diary into their regular routines?

11. How easy or difficult was it to navigate through the tasks? Did you use any type of "cheat sheet" or other written instructions to successfully complete the tasks?

12. Is there anything that you would like to let us know (e.g., suggestions for the future, clarification of any part of this study)?

\subsection{Data Analysis}

Both quantitative and qualitative information was obtained from participants in order to capture and analyze a broad range of data and feedback regarding the computer-based communication activities. This mixed methods design is known as the sequential transformative design approach where there are separate qualitative and quantitative data collection phases that are later integrated in order to understand the process [21] such as the user experience with the computer-based 
communication activities in this case. The qualitative data were analyzed using Track Changes and the highlighting function in Microsoft Word to organize codes and categories. Breaking down the qualitative data into smaller segments until larger themes arise was recommended in [22]. Examples of categories under each theme are shown in Table 3. Descriptive statistics (e.g., means and frequencies) were computed using SPSS version 19, whereas qualitative data were analyzed for content to supplement the quantitative data.

Table 3. Qualitative categories and themes.

\begin{tabular}{ccc}
\hline Accessibility & Awareness & Safety and Intrusion \\
\hline Finances & Eating & Privacy \\
Specificity & Routine & Security \\
Flexibility & Remembering & Emergency \\
Rural areas & Tech savvy & - \\
Timing & Socialization & - \\
\hline
\end{tabular}

\section{Results}

The results are reported in two sections. First, participants' performance and compliance rate in their usage for everyday health monitoring were examined in five domains (i.e., general computer skills and usage, overall perceived health, nutritional consumption, physical activity, and cognitive fatigue). Second, themes that emerged from the focus group discussion are also presented.

\subsection{Older Adults' Abilities and Compliance Rate in Health Monitoring}

After the ten-day period, further statistical analysis was conducted to evaluate older adults' abilities and the compliance rate in their usage for everyday health monitoring. Participants of this study reported an average rating of 2.88 on a 5-point scale on their confidence in computer skills, meaning that participants were somewhat uncertain about their skills in using a computer (Table 4). However, about a third of the participants stated they were somewhat confident in computer skills. In terms of computer usage, participants reported their computer usage ranged from once a month to every day to several times a day. Half of the sample indicated they used computers every day.

Table 4. Baseline computer skills.

\begin{tabular}{clcc}
\hline Category & Frequency & Percent \\
\hline \multirow{5}{*}{ General Computer Skill } & Very Confident (=5) & 0 & 0.0 \\
& Somewhat Confident $(=4)$ & 3 & 37.5 \\
& Neutral (= 3) & 2 & 25.0 \\
& Somewhat Uncertain $(=2)$ & 0 & 0.0 \\
& Very Uncertain (=1) & 3 & 37.5 \\
& Total & 8 & 100.0 \\
\hline \multirow{5}{*}{ Computer Usage } & Every day & 2 & 25.0 \\
& Several Times a Day & 4 & 50.0 \\
& Several Times a Week & 1 & 12.5 \\
& Once a Month & 1 & 12.5 \\
& Total & 8 & 100.0 \\
\hline
\end{tabular}

With regard to the compliance rate over the ten-day period, two users completed the 21 health diary questions every day (100\% compliance rate) and two other users completed the health diary questions less than $50 \%$ over the 10-day period. Specifically, all users completed the health diary questions on Day 1 (100\% compliance rate); however, the compliance rate dropped to $75 \%$ on Day 2, and the lowest compliance rate of $62.5 \%$ was reported on Day 3, 5, 6, and 10 (Figure 1). In addition, individual compliance rates can be found in Table 5 . 


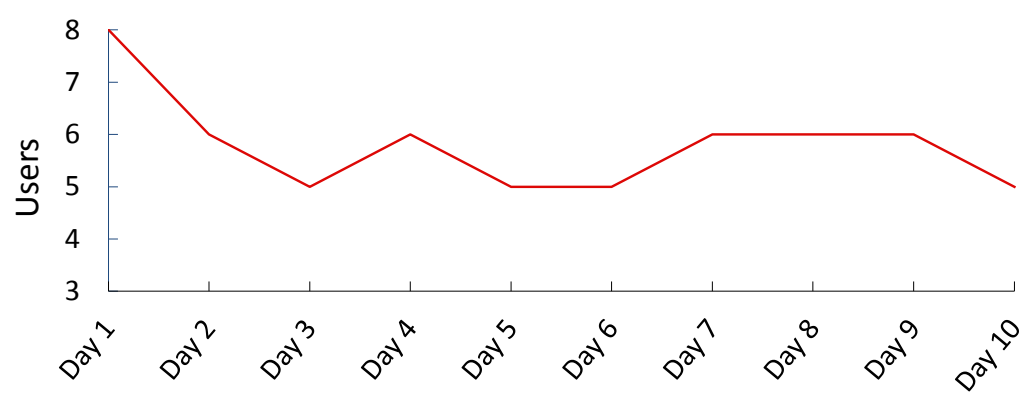

Figure 1. Number of users that completed the questionnaire each day.

Table 5. User compliance rates.

\begin{tabular}{ccccccccc}
\hline & User 1 & User 2 & User 3 & User 4 & User 5 & User 6 & User 7 & User 8 \\
\hline Compliance Rates & $90 \%$ & $90 \%$ & $40 \%$ & $100 \%$ & $80 \%$ & $100 \%$ & $70 \%$ & $10 \%$ \\
\hline
\end{tabular}

Based on the compliance rate, two groups were created: a high compliance rate group $(90 \%$ and above) and a low compliance rate group (below 90\%). Mean differences in computer skills, computer usage, and health at baseline between the two groups were examined through $t$-tests. However, no significant differences in the three variables were noted: $t(6)=0.27, p=0.80$ for computer skills, $t(6)=1.22, p=0.27$ for computer usage, or $t(6)=0.91, p=0.40$ for health at baseline.

\subsection{Three Themes from a Focus Group}

The ten-day pilot test was successful in that all participants who started the pilot test completed the ten-day testing period. This study also yielded interesting findings regarding older adult user experience with computer-based communication activities. Three total themes emerged from the focus group transcript: accessibility, awareness, and safety and intrusion.

\subsubsection{Accessibility}

This theme dealt with the accessibility from a number of different perspectives including environmental inclusion (e.g., rural environments) and financial accessibility (e.g., inexpensive technology will be used by more individuals). Participants were intrigued that Skype was free and felt it offered an additional benefit over cell phones. One participant noted, "A program that is free will be used." In addition, one participant suggested that a "fee scale" for healthcare services in which telehealth visits conducted using computer-based communication tools were cheaper than face-to-face visits could be advantageous. Another participant commented, "If it's [telehealth visits] lower [cost] than the actual visit, that's convenient." However, one participant went on to mention, "I thought professionals, if they wanted to use it for this type of thing [for telehealthcare], would need very good grade cameras."

Participants also felt that the check-in, daily diary, and Skype tasks were relatively easy to complete and use. A participant mentioned, "I did find out that the computer doesn't bite ... if you use it right." This sentiment seemed to illustrate the anxiety or hesitation that is sometimes associated with computers. Overall, after using computers over a ten-day period, participants seemed to possess more self-assurance. Although all participants did have their own computers, most individuals were only using their computers for basic tasks (e.g., playing games or checking email periodically). Only one participant had ever used Skype before. It was also apparent to the researchers that participants were much more at ease with the communication tools than they were at the beginning of the study. For example, researchers noted significant hesitancy and anxiety (e.g., concern that they might not complete the communication task successfully) on the day the programs were installed on participant computers. Participants also later stated how relatively easy the check-in task was to complete on the 
computer by indicating that it was "very simple and easy." Another participant stated, "No, I didn't have any trouble with the check-in."

Increased flexibility for check-in time periods was suggested by several participants. For example, one participant noted, "Our problem is from 10 a.m.-12 p.m. ... we didn't always get that [check-in] done." Other participants noted conflicting appointments or schedules that interfered with the check-in time frame. Furthermore, some participants felt the daily health diary could be more tailored to individual needs. For example, one participant responded, "I think that as the system would be refined ... questions would vary over time." Some participants seemed to note the importance of thinking about individual differences. The participant went on to explain:

"Not criticizing your question, but ... if you carried this thing a step further and it was being used by professionals, your questions wouldn't be of such a general nature and ultimately the questions would have to be refined to the person."

In regard to how detailed the daily health diary should be, one participant noted that some daily healthy diary questions may need to be more specific:

"Some will have quite the detailed questionnaire and others will have a relatively simple one. There is a lot of relativity as you move to one person to the other."

Participants also appreciated that the computer-based communication activities were relatively easy and quick to complete. Participants commented that the daily health diary took the longest and ranged anywhere from three to ten minutes to complete.

Although participants in this study were not living in a rural area, several individuals discussed the benefits for rural populations. One participant noted, "I think it would pertain more to people who are maybe more than maybe 100 miles away." Another participant commented, "And weather is important because in rural Iowa, winter weather snowy roads ... this would be advantageous."

\subsubsection{Awareness}

This theme was related to increased awareness of food and drink consumption, as well as increased awareness technology terms and opportunities for using technology. Participants appeared to be more aware of their own health when using the daily health diary as some questions asked about fruit and vegetable servings as well as liquid intake. For example, one participant noted, "It just reminded us to eat a little better." Similarly, an additional participant mentioned, "Your two fruits, two vegetables and 3 to 5 glasses or cups of water reminded us." Another participant noted, "... It did make me very much aware of the fluid intake."

One of the challenges associated with the computer-based communication activities were that "you had to remember to do it every day." For some, this requirement was challenging. Some participants reported difficulty remembering to complete the check-in, daily diary, and Skype tasks each day. Several participants noted they "forgot." However, one participant seemed to indicate that the tasks could soon become part of a routine. He stated, "If I just did it when I got up in the morning, there are all those things that you do routinely."

Participants also seemed to be more aware of available technology that might be useful to them and felt they became more knowledgeable about computer terms. One participant noted that "She ... our daughter ... was Skyping her husband in Washington, D.C. If they had said we were 'Skyping,' we would have thought, 'what on earth are they doing?' But we knew the language ... " Participants reported feeling satisfied that they were more knowledgeable about computer terminology (such as Skype) and were more aware of how such technology functioned. Skype also offered increased opportunities for socialization. A participant noted "I've made contact with relatives that I haven't seen for years. It's just like they're sitting across the table from you the way we sit and talk." 


\subsubsection{Safety and Intrusion}

This theme included discussion on increased safety due to technology tools as well as discussion on privacy and intrusion due to technology use. Participants mentioned that the computer-based communication tools would be helpful for safety reasons, particularly when professional staff members were away or unavailable. One participant mentioned that "[Staff members] need to keep in contact with me so [staff members] don't find me at the bottom of the stairs in a mess." An additional participant seemed to echo similar points when he indicated that "all the emergencies occur on weekends."

Participants seemed to acknowledge that check-in devices are important to ensuring the safety or older adults, especially those who reside alone or with little to no professional medical staff. One participant went on to further explain how useful check-in tasks are when he explained,

\footnotetext{
"But, uh, any kind of signal that we came up with [at the independent living apartment complex] ... either people didn't want us to be checking on them or they figured it wasn't a good enough plan and we've never done anything. And, generally speaking, we know what the next-door neighbor's doing or some person we are in touch with. But it failed once. I mean we lost somebody over a weekend..."
}

Another participant mentioned how beneficial a camera could be if one were to use Skype as part of telehealthcare. The participant said, "It seemed to me that the Skype would add a lot to that ... just seeing the individual, more than the telephone."

Initially, participants were less eager to use Skype to communicate with research assistants at the University. However, at the end of the research project participants thoroughly enjoyed the added benefit of being able to see the person they were communicating with during conversation. One participant noted, "I thought it was fun interacting with these students. I thought that was interesting." Another participant stated, "They [researcher assistants] weren't being intrusive." At the conclusion of the study, participants were eager to have the Skype software left on their computers for continued use. One participant noted, "You can take anything you want out of my computer, but leave Skype!"

\section{Discussion}

The purpose of this research was to implement computer-based communication activities to test with an older adult population and to evaluate the user experience of such tools by conducting a focus group following the research study. Previous research has indicated that the acceptability of monitoring technology has been understudied [21]. This study helped fill that gap by obtaining further information leading to the understanding of older adult acceptance levels of computer-based communication tools. For example, participants appreciated Skype and noted the benefits of using similar technologies for telehealthcare purposes in the future, especially for rural older adults who may find it difficult to travel to appointments. This study also simulated what it is like to have virtual contact with older adults (including the oldest old) and adds to the existing literature in the field of technology and aging. For example, previous research has noted the importance of developing e-health interventions to assist older adults in managing their physical and mental needs [22]. The three different computer-based communication activities used in the study contribute to the literature on older adults managing their health both physically and mentally. For example, the daily health diary required participants to record and track various health behaviors over the ten-day period. Previous research has noted that tracking and reminding participants about health behaviors aids in managing and making changes to overall health [23]. This study is also unique in that the mean age of participants was approximately 85 years of age. Little research assessing computer-based communication activities has implemented technology tools with the oldest old although recent studies have shown more older adults aged 65 years and older have used Internet to obtain health-related information [24]. This study adds to the relatively unknown research about the user experience with computer-based communication activities among the oldest old. 
Results from this study indicate that computer-based communication activities or health monitoring is a feasible option for older adults. Some participants were initially unsure about participating in the study and were concerned about the level of intrusiveness associated with the study. However, it was apparent during the focus group discussion that participants overcame their initial fears and relatively easily navigated the check-in, daily diary, and Skype tasks. This research also addresses previous concerns about older adult ability levels using technology for monitoring purposes [25]. Our findings indicate that older adults are able to successfully use computer-based communication activities despite initial concerns about monitoring and privacy.

\subsection{Design Best Practices}

In addition, it appears that part of the apparent success experienced by users may be due to researchers installing all software on participant computers, thereby providing one-click access. Offering support staff to install and demonstrate communication software also appears to be related to older adults finding the tools easy to use (i.e., researchers were available to answer any questions participants had about using the software). In addition, it was advantageous for users to have written instructions about navigating the different communication tools. Many of these designs are based on best practices for designing content for older adults [23]. Future researchers would do well to incorporate these items as well as other suggestions about making computer applications and content user-friendly.

In addition, it is important to point out the relatively inexpensive technology used for the purposes of this study. Adopting free or inexpensive programs for health monitoring or communication with healthcare providers is also more inclusive than programs with higher financial price tags. Such programs exclude a proportion of the population unable to afford such options. Although relatively simple in nature, check-in devices can give family members peace of mind from far away. Healthcare professionals would also easily be able to phone an older adult if he or she had not "checked-in" that day. Such simple measures and applications can be beneficial and efficient for a wide range of individuals (e.g., older adults, family members, and healthcare professionals). Implementing computer-based communication activities with "real" healthcare staff involved may also prove useful in determining which design elements both older adults and healthcare professionals prefer.

\subsection{Limitations}

Limitations of this study are that only participants residing in one older adult independent apartment complex were included. It is possible that older adults residing in different types of communities and geographic locations may have had different experiences using the computer-based communication activities. It is interesting to note that the mean age of participants was approximately 85 years of age. Therefore, testing out technology tools with a younger sample of older adults may yield different perspectives. Additionally, this study was a pilot assessment; future opportunities exist in creating a randomized controlled study for comparisons with multiple conditions.

\subsection{Implications and Future Research}

Technology tools should continue to be tested with a geographically and demographically wider range of older adult populations in order to assess older adult preferences and overall effectiveness. The feasibility of using computer-based communication activities with older adults residing in other types of communities (e.g., assisted living) should also be conducted. Additionally, conducting studies assessing older adults using computer-based communication activities longer than a ten-day period should be conducted in the future. Compliance rates may vary with extended time intervals. Finally, compliance rates may also have been impacted by asking the same questions on the daily health diary each day. It is possible that if the individual were asked unique and tailored questions (based on previous answers), compliance rates may have been higher. Asking fewer questions each 
day may have also impacted compliance rates and made it easier for participants to complete each day. Several participants also noted that they were unable to call in during the Skype hours due to prior conflicts or forgetfulness. It is possible that, had the Skype phone call hours been extended, compliance rates would have been higher. If a longer study had been conducted, trends in decision-making could have been assessed (e.g., the extent to which participants changed their eating and exercise patterns) as a result of participating in the study and completing the daily health diary for a prolonged period of time. It is also likely that participants would have become even more adept at their computer skills with additional practice and a daily requirement in place. Lastly, a longer study would have been able to more accurately assess compliance (e.g., whether or not participants were more or less likely to complete tasks over an extended period of time).

As a result of this study, we believe using telehealthcare technology in the future with older adults can be a feasible option for monitoring and assessing the well-being of older adults. The older adults in this study enjoyed the computer-based communication activities and even reported that they were more aware of their health (e.g., aware of how many servings of fruits and vegetables to eat, how much fluid to drink) due to the daily diary tool. Participants also found the Skype tool to be useful when checking in with research assistants and seemed to enjoy the built-in socialization component. Overall, participants appeared comfortable and confident about technology use by the end of the study (e.g., eagerness to monitor their own health behaviors and enjoyment in using the computer prototypes). Finally, older participants became more informed and aware of what technology can do for them in terms of healthcare and social engagement.

While the telehealthcare field is a new area, this study advanced what is known by testing three computer-based communication activities and obtaining user feedback and metrics for each tool. Some older adults may be able to complete all three steps of the computer-based activities seamlessly, whereas others may experience more difficulty. As the medical community becomes more technologically advanced, older adults may need to have increased contact with technology to receive healthcare services. Therefore, continued studies need to address older adult technology use and compliance.

Acknowledgments: We would like to thank Hen-I Yang for his assistance setting up and assisting with data collection.

Author Contributions: Melinda Heinz assisted with collecting Skype and focus group data as well as data analysis and contributed to writing the paper. Jinmyoung Cho analyzed quantitative data and assisted with the writing of the paper. Norene Kelly assisted with data analysis and the writing of the paper. Wen-Hua Hsieh assisted with collecting Skype data and setting up data collection procedures. Joan Blaser assisted with collecting Skype data and focus group data analysis. Peter Martin conceived and designed the study, collected data, and oversaw data analysis. Johnny Wong conceived and designed the study and designed data collection tools. Warren Franke conceived and designed the study.

Conflicts of Interest: The authors declare no conflict of interest.

\section{References}

1. Burdick, D.C., Kwon, S., Eds.; Gerontechnology: Research and Practice in Technology and Aging; Springer: New York, NY, USA, 2004; pp. xxv-xxvii.

2. Rogers, E. Diffusion of Innovations, 5th ed.; Free Press: New York, NY, USA, 2003.

3. Czaja, S.J.; Lee, C. The impact of aging on access to technology. Univers. Access Inf. Soc. 2007, 5, 341-349. [CrossRef]

4. Demeris, G.; Rantz, M.J.; Aud, M.A.; Marek, K.D.; Tyrer, H.W.; Skubic, M.; Hussam, A.A. Older adults' attitudes towards and perceptions of "smart home" technologies: A pilot study. Med. Inf. Internet Med. 2004, 29, 87-94. [CrossRef] [PubMed]

5. Magnusson, L.; Hanson, E. Supporting frail older people and their family carers at home using information and communication technology: Cost analysis. J. Adv. Nurs. 2005, 51, 645-657. [CrossRef] [PubMed]

6. Wang, A.; Redington, L.; Steinmetz, V.; Lindeman, D. The ADOPT model: Accelerating diffusion of proven technologies for older adults. Ageing Int. 2011, 36, 39-45. [CrossRef] 
7. Pew, R., Van Hemel, S., Eds.; Technology for Adaptive Aging; National Academy Press: Washington, DC, USA, 2004.

8. Cutler, S.J. Ageism and technology. Generations 2005, 29, 67-72.

9. Eisma, R.; Dickinson, A.; Goodman, J.; Syme, A.; Tiwari, L.; Newell, A.F. Early user involvement in the development of information technology-related products for older people. Univers. Access Inf. Soc. 2004, 3, 131-140. [CrossRef]

10. Charness, N.; Boot, W.R. Aging and information technology use: Potential and barriers. Curr. Dir. Psychol. Sci. 2009, 18, 253-258. [CrossRef]

11. de Moraes Barros, G.D.V. Falls in elderly people. Lancet 2006, 367, 729-730. [CrossRef]

12. Scanaill, C.N.; Garattini, C.; Greene, B.R.; McGrath, M.J. Technology innovation enabling falls risk assessment in a community setting. Ageing Int. 2011, 36, 217-231. [CrossRef] [PubMed]

13. Kannus, P.; Sievanen, H.; Palvanen, M.; Jarvinen, T.; Parkkari, J. Prevention of falls and consequent injuries in elderly people. Lancet 2005, 366, 1885-1893. [CrossRef]

14. Dishman, E.; Matthews, J.; Dunbar-Jacob, J. Everyday health: Technology for adaptive aging. In Technology for Adaptive Aging; Pew, R.W., Van Hemel, S.B., Eds.; National Academy Press: Washington, DC, USA, 2004; pp. 179-208.

15. Fallon, L.F., Jr.; Awosika-Olumo, A.; Fulks, J.S. Factors related to accidents and falls among older individuals. Traumatology 2002, 8, 205-210. [CrossRef]

16. Nehmer, J.; Becker, M.; Kleinberger, T.; Pruckner, S. Electronic emergency safeguards: Sensor-based detection and prevention of critical health conditions. GeroPsych 2010, 23, 91-98. [CrossRef]

17. Dishman, E. Inventing wellness systems for aging in place. Computer 2004, 37, 34-41. [CrossRef]

18. Rogers, W.A.; Mayhorn, C.B.; Fisk, A.D. Technology in everyday life for older adults. In Gerontechnology: Research and Practice in Technology and Aging; Burdick, D.C., Kwon, S., Eds.; Springer: New York, NY, USA, 2004; pp. 3-17.

19. Virzi, R.A. Refining the test phase of usability evaluation: How many subjects is enough? J. Hum. Factors Ergon. Soc. 1992, 34, 457-468.

20. Merriam, S.B. Qualitative Research, a Guide to Design and Implementation; Jossey-Bass: San Francisco, CA, USA, 2009.

21. Terrell, S. Mixed-methods research methodologies. Qual. Rep. 2012, 17, 254-280.

22. Creswell, J.W. Research Design: Qualitative, Quantitative, and Mixed Methods Approaches; Sage Publications Ltd.: Thousand Oaks, CA, USA, 2008.

23. Klasnja, P.; Consolvo, S.; McDonald, D.W.; Landay, J.A.; Pratt, W. Using mobile and personal sensing technologies to support health behavior change in everyday life: Lessons learned. AMIA Annu. Symp. Proc. 2009, 2009, 338-342. [PubMed]

24. Wright, P. The internet's potential for enhancing healthcare. Gerontechnology 2012, 11, 35-45. [CrossRef]

25. Preschl, B.; Wagner, B.; Forstmeier, S.; Maercker, A. E-health interventions for depression, anxiety disorder, dementia, and other disorders in adults: A review. J. CyberTher. Rehabil. 2011, 4, 371-385.

(C) 2016 by the authors; licensee MDPI, Basel, Switzerland. This article is an open access article distributed under the terms and conditions of the Creative Commons Attribution (CC-BY) license (http://creativecommons.org/licenses/by/4.0/). 